\title{
SageDB and NetAccel
}

ADRIAN COLYER

LEARNED MODELS WITHIN THE DATABASE SYSTEM; NETWORKACCELERATED QUERY PROCESSING

For the last few years, we have been avid followers of Adrian Colyer's blog, The Morning Paper. We read and admire The Morning Paper for the same reasons we began Queue-it delivers practical ideas from academia to the computing practitioner in a useful, no-nonsense fashion. We know this approach works and our ever-growing readership supports this as well. In The Morning Paper, Adrian distills academic papers in a way that makes them naturally accessible to the general practitioner. It should be read by every Queue reader and we are delighted to welcome Adrian and The Morning Paper to our publication.

- Stephen Bourne and Theo Schlossnagle Queue editorial board co-chairs

The CIDR (Conference on Innovative Data Systems Research) runs once every two years, and luckily for us 2019 is one of those years. I've selected two papers from this year's conference (held January 13-16) that highlight bold and exciting directions for data systems.

In "SageDB: A Learned Database System," the authors explore the potential of embracing machine learning within the very core of a database system: learned models that pervade the algorithms and data structures of the system itself. It hints at an interesting future for systems software in general, with machine-learned components augmenting 
and adapting systems to fit their workloads and execution environments.

My second choice is "The Case for Network-accelerated Query Processing." With programmable switches we have a whole new option for processing placement in distributed systems-we can now perform certain tasks "in the wire!" With NetAccel, the authors show how parts of the query processing for an MPP (massively parallel processing) database can be done at line rate in packet-processing hardware. The resulting performance improvements are hard to ignore.

For the complete column, go to https:/lqueue.acm.orgl demo/TMPlindex.cfm

Adrian Colyer is a venture partner with Accel in London, where it's his job to help find and build great technology companies across Europe and Israel. (If you're working on an interesting technology-related business he would love to hear from you: you can reach him at acolyer@accel.com.] Prior to joining Accel, he spent more than 20 years in technology companies, including CTO roles at Pivotal, VMware, and SpringSource.

Copyright $@ 2019$ held by ownerlauthor. Publication rights licensed to ACM. Reprinted with permission from https:I/blog.acolyer.org 